\title{
Some Remarks on Microhypoelliptic Operators of Infinitely Degenerate Type
}

Dedicated to Professor Shigetake Matsuura on his sixtieth birthday

By

\section{Tatsushi MORIOKA*}

\section{$\S 1$. Introduction and Results}

Let $P$ be a linear partial differential operator in $\boldsymbol{R}^{n}$ with $C^{\infty}$ coefficients and $z$ be a point in $T^{*}\left(\boldsymbol{R}^{n}\right) . \quad P$ is said to be microhypoelliptic at $z$ if $z \notin W F(P u)$ implies $z \notin W F u$ for any $u \subseteq \mathscr{D}^{\prime}$. For $z=\left(x^{0}, \xi^{0}\right) \in T^{*}\left(\boldsymbol{R}^{n}\right), z \notin W F u$ if there exists a function $\phi \in C_{0}^{\infty}\left(\boldsymbol{R}^{n}\right)$ with $\phi=1$ near $x^{0}$ and a conic neighborhood $\Gamma$ of $\xi^{0}$ such that for every positive $M$ we have with a constant $C_{M}$

$$
|\widehat{\phi u}(\xi)| \leqq C_{M}(1+|\xi|)^{-M}, \quad \xi \in \Gamma .
$$

Here $\hat{v}$ denotes the Fourier transform of $v$.

In this paper, we shall study the microhypoellipticity of the following operator in $\boldsymbol{R}^{n}=\boldsymbol{R}_{x}^{n_{1}} \times \boldsymbol{R}_{y}^{n_{2}}$

$$
L=a\left(x, y, D_{x}\right)+g(x) b\left(x, y, D_{y}\right)
$$

satisfying the following conditions. (Throughout this paper, the coefficients of differential operators are assumed to be $C^{\infty}$.)

(A.1) $g(0)=0$ and $g(x)>0$ for $x \neq 0$.

(A.2) $a\left(x, y, D_{x}\right)$ is a differential operator of order $2 l$ and

$$
\operatorname{Re} a(x, y, \xi) \geqq C_{1}|\xi|^{2 l}
$$

holds for sufficiently large $|\xi|$.

(A.3) $b\left(x, y, D_{y}\right)$ is a differential operator of order $2 m$ and

$$
\operatorname{Re} b(x, y, \eta) \geqq C_{2}|\eta|^{2 m}
$$

Communicated by T. Kawai, December 25, 1990. Revised March 5, 1991 and May 14, 1991.

1991 Mathematics Subject Classifications : 35H05.

* Department of Mathematics, Osaka University, Osaka Japan. 
holds for sufficiently large $|\eta|$. Here $C_{1}, C_{2}$ are positive constants and $l, m$ are positive integers.

In Morioka [5], we have shown that $L$ is hypoelliptic, i.e.,

$$
\text { sing supp } u=\operatorname{sing} \operatorname{supp} L u \quad \text { for } u \in \mathscr{D}^{\prime}
$$

if (A.1)-(A.3) are satisfied. This is the improvement of Morimoto [3]. Actually, in [3] the following condition $(G)$ was required to show (1.2) in case of $m \geqq 2$ :

(G) There exist constants $C$ and $\tau(0<\tau<1 /(2 l m+2 m-2 l))$ such that

$$
\left|D_{x}^{\alpha} g(x)\right| \leqq C g(x)^{1-\tau|a|} \quad \text { near } x=0,
$$

for any multi-index $\alpha$ with $|\alpha| \leqq 2 l m+2 m-2 l$.

Our main result concerning the microhypoellipticity of $L$ is the following:

Theorem 1. Let $z=\left(x^{0}, y^{0} ; \xi^{0}, \eta^{0}\right)$ be a point in $T^{*}\left(\mathbb{R}^{n}\right)\left(x^{0}, \xi^{0} \in \boldsymbol{R}^{n_{1}}\right.$ and $y^{0}$, $\left.\eta^{0} \in \boldsymbol{R}^{n_{2}}\right)$ with $\left|\eta^{0}\right| \neq 0$. Let $L$ be the operator (1.1) satisfying (A.1)-(A.3).

(i) In the case where $l \geqq m, L$ is microhypoelliptic at $z$.

(ii) In the case where $l<m, L$ is still microhypoelliptic at $z$ if $g(x)$ satisfies the following condition.

(A.4) There exist constants $C$ and $\tau(0<\tau<1 /(2 m-2 l))$ such that

$$
\left|D_{x}^{\alpha} g(x)\right| \leqq C g(x)^{1-\tau|a|} \quad \text { near } x=0,
$$

for any multi-index $\alpha$ with $|\alpha| \leqq 2 m-2 l$.

The condition (A.4) is always satisfied whenever $(G)$ holds for $g(x)$. If $n_{1}=1, x \in \boldsymbol{R}$ and $g(x)=x^{2 k}$, (A.4) is equivalent to the condition $k>m-l$. By Parenti-Rodino [6], it is known that if $0<k \leqq m-l$, the hypoelliptic operator $D_{x}^{2 l}+x^{2 k} D_{y}^{2 m}$ (in $\boldsymbol{R}^{2}$ ) is not microhypoelliptic. Roughly speaking, Theorem 1-(ii) implies that the large vanishing order of $g(x)$ makes $L$ be microhypoelliptic at $z$, under the assumption concerning the magnitudes of derivatives of $g(x)$. We wish to consider the case where $g(x)$ vanishes infinitely at the origin (i.e., $D^{a} g(0)=0$ for any $\alpha$ ) and does not satisfy (A.4) in the forthcoming paper [4].

Now we explain our idea. The main tool of the proof of Theorem 1 is the following proposition.

Proposition 1.1. Let $L$ be the operator (1.1) satisfying (A.1)-(A.3). Assume moreover the following condition.

(A.5) $L$ is microhypoelliptic at any $\tilde{z}=(\tilde{x}, \tilde{y} ; \tilde{\xi}, \tilde{\eta}) \in T^{*}\left(\mathbb{R}^{n}\right)$ with $|\tilde{\xi}||\tilde{\eta}| \neq 0$.

Then $L$ is also microhypoelliptic at any $z=\left(x^{0}, y^{0} ; 0, \eta^{0}\right)$ with $\left|\eta^{0}\right|=1$.

In the case where $l \geqq m$, Proposition 1.1 completes the proof of Theorem 
1-(i). It is clear that (A.5) are satisfied without any additional conditions if $l \geqq m$. Indeed, if we choose a conic neighborhood $V$ of $(\tilde{x}, \tilde{y} ; \tilde{\xi}, \tilde{\eta})$ such that $|\xi| \sim|\eta|$ for $(x, y ; \xi, \eta) \in V$, we have with a positive constant $C$

$$
\operatorname{Re} p(x, y, \xi, \eta) \geqq C\left(1+|\xi|^{2}+|\eta|^{2}\right)^{l} \quad \text { for }(x, y ; \xi, \eta) \in V .
$$

Here we supposed that $|\tilde{\xi}||\tilde{\eta}| \neq 0$ and $p$ is the symbol of $L$. In the case where $l<m$, we shall see that (A.5) are satisfied under the additional condition (A.4). We give the proof of Proposition 1.1 in Section 1, by refining microlocally Section 3 of [5]. In Section 2, we show that (A.5) follows from (A.1)-(A.4). (A.4) is used only to see that (A.5) are satisfied in the case where $l<m$.

\section{$\S 2$. Proof of Proposition 1.1}

Let $L$ be the operator (1.1) satisfying (A.1)-(A.3) and (A.5). Since $L$ is semi-elliptic in $\left\{(x, y) \in \boldsymbol{R}^{n}: x \neq 0\right\}, L$ is microhypoelliptic at $z=\left(x^{0}, y^{0} ; \xi^{0}, \eta^{0}\right) \in$ $T^{*}\left(\boldsymbol{R}^{n}\right)$ if $\left|x^{0}\right| \neq 0$. See also [2, Chapter 2, §5]. So we restrict our consideration to the case where $x^{0}=0$. Our purpose is to show that $z \notin W F u$ if $z \notin$ $W F(L u)$, where $z=\left(0, y^{0} ; 0, \eta^{0}\right)$ with $\left|\eta^{0}\right|=1$. We may assume $u \in \mathcal{E}^{\prime}$. Since the proof is long, we devided it into three steps. (A.5) are used in Step 1 and (A.1)-(A.3) are in Step 2.

\section{(Step 1)}

We choose two smooth functions $\gamma(\xi, \eta)$ and $\alpha(\eta)$ as follows. Let $\Gamma_{1}$ and $\Gamma_{2}$ be small cones in $\boldsymbol{R}^{n}$ such that $\left(0, \eta^{0}\right) \equiv \Gamma_{1}$ and $\Gamma_{1} \Subset \Gamma_{2}$. The support of $\gamma$ is in $\Gamma_{2}$ and $\gamma=1$ in $\Gamma_{1} \cap\left\{|\xi|^{2}+|\eta|^{2} \geqq 1\right\}$. Let $F_{1}$ and $F_{2}$ be small cones in $\boldsymbol{R}_{\eta}^{n_{2}}$ such that $\eta^{0} \in F_{1}, F_{1} \Subset F_{2}$ and $\left\{(0, \eta) \in \boldsymbol{R}^{n}: \eta \in F_{2}\right\} \Subset \Gamma_{1}$. The support of $\alpha$ is in $F_{2}$ and $\alpha=1$ in $F_{1} \cap\{|\eta| \geqq 1\}$. Moreover $\gamma \in S_{1,0}^{0}\left(\boldsymbol{R}^{n}\right)$ and $\alpha \in S_{1,0}^{0}\left(\boldsymbol{R}^{n_{2}}\right)$.

Next, take $\dot{\phi}_{1}(x, y) \in C_{0}^{\infty}\left(\boldsymbol{R}^{n}\right)$ with $\dot{\phi}_{1}=1$ near $(x, y)=\left(0, y^{0}\right)$. Assume that $z \notin W F(L u)$. Then we have

$$
\gamma\left(D_{x}, D_{y}\right)\left(\phi_{1} L u\right) \in H^{\infty}
$$

by taking the support of $\phi_{1}(x, y)$ and $\gamma(\xi, \eta)$ sufficiently small. Take moreover $\phi(x, y)=\chi(x) \phi(y)$ with $\chi(x) \in C_{0}^{\infty}\left(\boldsymbol{R}^{n_{1}}\right), \chi=1$ near $x=0, \phi(y) \in C_{0}^{\infty}\left(\boldsymbol{R}^{n_{2}}\right), \phi=1$ near $y=y^{0}$ and $\phi \Subset \phi_{1}$, i.e., the support of $\phi$ is contained in a neighborhood of the closed set where $\phi_{1}=1$. Then from (2.1) we have

$$
\phi(x, y) \gamma\left(D_{x}, D_{y}\right) L u \in H^{\infty} .
$$

Moreover we see that

$$
\alpha\left(D_{y}\right)\left(\phi L \gamma\left(D_{x}, D_{y}\right) u\right)=\alpha(\phi \gamma L u)-\alpha(\dot{\phi}[\gamma, L] u) .
$$

On one hand, the asymptotic expansion gives 


$$
\left[\gamma\left(D_{x}, D_{y}\right), L\right] u \sim \sum_{|\mu| \geqq 1}(\mu !)^{-1} L_{(\mu)} \gamma^{(\mu)}\left(D_{x}, D_{y}\right) u,
$$

where $L_{(\mu)}=\left(D_{x, y}^{\mu} L\right)\left(x, y, D_{x}, D_{y}\right)$ and $\gamma^{(\mu)}\left(D_{x}, D_{y}\right)=\left(\partial_{\xi, \eta}^{\mu} \gamma\right)\left(D_{x}, D_{y}\right)$. On the other hand, we may assume

$$
\alpha\left(D_{y}\right)\left(\phi L_{(\mu)} \gamma^{(\mu)}\left(D_{x}, D_{y}\right) u\right) \in H^{\infty}
$$

for $|\mu| \geqq 1$. The reason is that $L$ satisfies (A.5) (i.e., $L$ is microhypoelliptic at any $\tilde{z}=(\tilde{x}, \tilde{y} ; \tilde{\xi}, \tilde{\eta}) \in T^{*}\left(\boldsymbol{R}^{n}\right)$ with $\left.|\tilde{\xi}||\tilde{\eta}| \neq 0\right)$ and $\gamma^{(\mu)}(\xi, \eta)=0$ on $\Gamma_{1} \cap$ $\left\{|\xi|^{2}+|\eta|^{2} \geqq 2\right\}$ in case of $|\mu| \geqq 1$. Notice that $\left\{(0, \eta) \in \mathbb{R}^{n}: \eta \in \operatorname{supp} \alpha\right\} \Subset \Gamma_{1}$. Therefore it follows that $\alpha(\phi[\gamma, L] u) \in H^{\infty}$. Thus in view of (2.2) and (2.3), we have

$$
\alpha\left(D_{y}\right)\left(\phi L \gamma\left(D_{x}, D_{y}\right) u\right) \in H^{\infty}
$$

(Step 2)

From (2.5) we see that

$$
\alpha \phi L \chi \gamma u=\alpha \phi L \gamma u+\alpha \phi[L, \chi] \gamma u .
$$

Recall that $\phi(x, y)=\chi(x) \psi(y)$. Noticing that the support of $[L, \chi] \gamma u$ does not intersect $\{x=0\}$, it follows that

$$
\alpha\left(D_{y}\right)(\psi L v) \Subset H^{\infty},
$$

where $v=\chi \gamma\left(D_{x}, D_{y}\right) u$.

Now let $\Phi(t) \in C_{0}^{\infty}\left(\boldsymbol{R}^{n_{2}}\right)$ be a non-negative function satisfying

and

$$
\Phi(t)=1 \quad \text { in }\left\{t:|t| \leqq r^{\prime}\right\}
$$

$$
\Phi(t)=0 \quad \text { in }\{t:|t| \geqq r\} .
$$

Here we supposed that $0<r^{\prime}<r<1$.

We define $\alpha_{n}(\eta)$ and $\beta(y)$ as follows :

$$
\alpha_{n}(\eta)=\Phi\left(\frac{\eta}{n}-\eta^{0}\right), \quad \beta(y)=\Phi\left(y-y^{0}\right),
$$

where $n$ is a positive integer.

Choose $r>0$ sufficiently small such that $\operatorname{supp} \alpha_{n} \Subset F_{1}$ and $\beta \Subset \psi$. Then we have the following lemma.

Lemma 2.1. If we fix $p$ and $q$,

$$
\left\|\alpha_{n}^{(p)}\left(D_{y}\right)\left(\beta_{(q)} L v\right)\right\|_{L^{2}\left(R^{n}\right)}=O\left(n^{-s}\right)
$$

holds for any positive number s. Here $\alpha_{n}^{(p)}$ and $\beta_{(q)}$ denote $\partial_{\eta}^{p} \alpha_{n}$ and $D_{y}^{q} \beta$, respectively.

Remark. In general, $c_{n}=O\left(n^{-k}\right)$ means that there exists a constant $B$ such 
that $\left|c_{n}\right| \leqq B n^{-k}$, when $n$ is large.

Proof of Lemma 2.1. For the sake of simplicity, we restrict our consideration to the case where $p=q=0$ since the proof in the other case is similar. Let us take $w=L v$ and consider the following estimate:

$$
\begin{aligned}
& n^{2 s}\left\|\alpha_{n} \beta w\right\|^{2}=\left\|n^{s} \alpha_{n}(\eta)(\widehat{\beta w})(\xi, \eta)\right\|^{2} \\
& \leqq \text { const. } \| \alpha_{n}(\eta)(\widehat{\beta w})(\xi, \eta)\left\langle\eta^{\prime} \|^{2} \leqq \text { const. } \iint_{F_{1}}|\widehat{\beta w}(\xi, \eta)|^{2}\langle\eta\rangle^{2 s} d \xi d \eta,\right.
\end{aligned}
$$

where $\langle\eta\rangle=\left(1+|\eta|^{2}\right)^{1 / 2}$. Recall that $c^{-1} n \leqq|\eta| \leqq c n$ for $\eta \in \operatorname{supp} \alpha_{n}$. Since $\alpha=1$ on $F_{1} \cap\{|\eta| \geqq 1\}$, we obtain the desired estimate in view of (2.7). Q.E.D.

Lemma 2.2. It holds that

$$
\left\|\alpha_{n}\left(D_{y}\right)(\beta v)\right\|_{L^{2}\left(\boldsymbol{R}^{n}\right)}=O\left(n^{-s}\right)
$$

for any positive number s.

Proof of Lemma 2.2. Our arguments in the proof of the above lemma is quite analogous to those in Section 3 of Morioka [5]. So we refer to [5] for some calculus which we omit.

Now take $h_{n, p, q}=\alpha_{n}^{(p)} \beta_{(q)} L v$ and $v_{n, p, q}=\alpha_{n}^{(p)} \beta_{(q)} v$. Then the asymptotic expansion gives

$$
\begin{aligned}
& \operatorname{Re}\left(L v_{n, p q}, v_{n, p, q}\right)_{L 2\left(\boldsymbol{R}^{n}\right)} \\
= & \operatorname{Re}\left(-\left[\alpha_{n}^{(p)} \beta_{(q)}, L\right] v+h_{n, p, q}, v_{n, p, q}\right)_{L 2\left(\boldsymbol{R}^{n}\right)} \\
= & \operatorname{Re} \sum_{k=1}^{5} I_{k}
\end{aligned}
$$

with

$$
\begin{aligned}
& I_{1}=-\sum_{1 \leqq||^{\prime}+\nu|\leqq N-| p+q \mid}(-1)^{|\nu|}(\nu !)^{-1}(\mu !)^{-1}\left(g(x) b_{(\mu)}^{(\nu)} v_{n, p+\mu, q+\nu}, v_{n, p, q}\right), \\
& I_{2}=-\left(g(x) r_{n, p, q}^{N} v, v_{n p q}\right), \\
& I_{3}=-\sum_{1 \leqq|\mu| \leqq N-|p+q|}(\mu !)^{-1}\left(a_{(\mu)} v_{n, p+\mu, q}, l_{n, p, q}\right), \\
& I_{4}=-\left(\tau_{n, \mu, q}^{N} v, v_{n, p q}\right), \\
& I_{5}=\left(h_{n, p, q}, v_{n, p, q}\right),
\end{aligned}
$$

where $N$ is a large integer whose definition will be given later.

Remark. $\left(\left[\alpha_{n}^{(p)} \beta_{(q)}, a\right] v, v_{n, p, q}\right)=I_{3}+I_{4}$ and $\left(\left[\alpha_{n}^{(p)} \beta_{(q)}, g(x) b\right] v, v_{n, p, q}\right)=I_{1}+I_{2}$.

Now we can estimate $\left|I_{1}\right|$ and $\left|I_{3}\right|$ as follows, by using the Garding's inequality with respect to $y$ and $x$ variable, respectively. 


$$
\begin{aligned}
\left|I_{1}\right| \leqq & \sum_{1 \leqq|l|+\nu|\leqq N-| p+q \mid}\left\{K^{-1}\left(C_{1} \operatorname{Re}\left(g(x) b v_{n, p, q}, v_{n, p, q}\right)+C_{2}\left\|v_{n, p, q}\right\|^{2}\right)\right. \\
& \left.+K n^{-2|\nu|}\left(C_{3} \operatorname{Re}\left(g(x) b v_{n, p+\mu, q+\nu}, v_{n, p+p, q+\nu}\right)+C_{4}\left\|v_{n, p+\mu, q+\nu}\right\|^{2}\right)\right\} \\
\left|I_{3}\right| \leqq & \sum_{1 \leqq|,| \leqq N-|p+q|}\left\{K^{-1}\left(C_{5} \operatorname{Re}\left(a v_{n, p, q}, v_{n, p, q}\right)+C_{6}\left\|v_{n, p, q}\right\|^{2}\right)\right. \\
& \left.+K\left(C_{7} \operatorname{Re}\left(a v_{n, p+\mu, q}, v_{n, p+\mu, q}\right)+C_{8}\left\|v_{n, p+\mu, q}\right\|^{2}\right)\right\} .
\end{aligned}
$$

Here each $C_{3}$ are positive constants. We estimate $\left|I_{2}\right|,\left|I_{4}\right|$ and $\left|I_{5}\right|$ in the following way.

$$
\begin{aligned}
& \left|I_{2}\right| \leqq K\left\|r_{n, p, q}^{N} v\right\|^{2}+K^{-1}\left\|v_{n, p, q}\right\|^{2} \\
& \left|I_{4}\right| \leqq K\left\|\tau_{n, p, q}^{N} v\right\|^{2}+K^{-1}\left\|v_{n, p, q}\right\|^{2} \\
& \left|I_{5}\right| \leqq K\left\|h_{n, p, q}\right\|^{2}+K^{-1}\left\|v_{n, p, q}\right\|^{2} .
\end{aligned}
$$

Remark. In (2.9)-(2.13), $K$ is an arbitrary positive constant and each $C$, are independent of the choice of $K$.

Take $c_{p q}^{n}=n^{(1 / 2)(|p|-|q|)}$. Then for any $s>0$ there exists a large integer $N$ such that

$$
\left\|c_{p q}^{n} r_{n, p, q}^{N} v\right\|=O\left(n^{-s}\right)
$$

and

$$
\left\|c_{p q}^{n} \tau_{n, p, q}^{N} v\right\|=O\left(n^{-s}\right)
$$

hold for $|p+q| \leqq N$.

Take $w_{n, p, q}=c_{p q}^{n} v_{n, p, q}$ and observe that $c_{p q}^{n}=n^{-(1 / 2)(|\mu|-|\nu|)} c_{p+\mu, q+\nu .}^{n}$ Let $s>0$ be a given number. We choose $N$ such that (2.14) and (2.15) hold. Then from (2.8)-(2.13) we have for any $\varepsilon>0$

$$
\begin{aligned}
& \quad \frac{1}{2} \operatorname{Re}\left(a w_{n, p, q}, w_{n, p, q}\right)+\frac{1}{2} \operatorname{Re}\left(g(x) b w_{n, p, q}, w_{n, p, q}\right) \\
& \leqq \\
& K_{1 \leqq|\mu+\nu| \leqq N-|p+q|} n^{-|\mu+\nu|}\left(C_{9} \operatorname{Re}\left(g(x) b w_{n, p+\mu, q+\nu}, w_{n, p+\mu, q+\nu}\right)\right. \\
& \left.\quad+C_{10}\left\|w_{n, p+\mu, q+\nu}\right\|^{2}\right)+K \sum_{1 \leqq|\mu| \leqq N-|p+q|} n^{-|\mu|}\left(C_{11} \operatorname{Re}\left(a w_{n, p+\mu, q}, w_{n, p+\mu, q}\right)\right. \\
& \left.\quad+C_{12}\left\|w_{n, p+\mu, q}\right\|^{2}\right)+\varepsilon\left\|w_{n, p, q}\right\|^{2}+K\left\|c_{p q}^{n} h_{n, p, q}\right\|^{2}+O\left(n^{-2 s}\right),
\end{aligned}
$$

by taking $K$ sufficiently large. 
Summing up the both sides of $(2.16)$ with respect to $(p, q)$ satisfying $|p+q|$ $\leqq N$, then we see that

$$
\begin{aligned}
& \frac{1}{2} A_{N, n}+\frac{1}{2} B_{N, n} \\
\leqq & K C_{13} n^{-1} A_{N, n}+K C_{14} n^{-1} B_{N, n}+2 \varepsilon S_{N, n}+K T_{N, n}+O\left(n^{-2 s}\right),
\end{aligned}
$$

where

$$
\begin{aligned}
& A_{N, n}=\sum_{|p+q| \leqq N} \operatorname{Re}\left(a w_{n, p, q}, w_{n, p, q}\right), \\
& B_{N, n}=\sum_{|p+q| \leqq N} \operatorname{Re}\left(g(x) b w_{n, p, q}, w_{n, p, q}\right), \\
& S_{N, n}=\sum_{|p+q| \leqq N}\left\|w_{n, p, q}\right\|^{2}, \\
& T_{N, n}=\sum_{|p+q| \leqq N}\left\|c_{p q}^{n} h_{n, p, q}\right\|^{2} .
\end{aligned}
$$

By Gårding's inequality with respect to $y$ variable, $B_{N, n} \geqq 0$ holds for sufficiently large $n$. In view of Lemma 2.1, it holds that $T_{N, n}=O\left(n^{-2 s}\right)$. Therefore we have

$$
\frac{1}{4} A_{N, n} \leqq \varepsilon S_{N, n}+O\left(n^{-2 s}\right) .
$$

If we choose the support of $\chi(x)$ sufficiently small, then we have

$$
A_{N, n} \geqq \delta S_{N, n}
$$

for some $\hat{\delta}=0$. See Lemma 3.2 in Morioka [5]. Therefore we see that

$$
S_{N, n}=O\left(n^{-2 s}\right) \text {. }
$$

Recall that $\varepsilon$ can be any positive number. Since $\left\|\alpha_{n} \beta v\right\|^{2} \leqq S_{N, n}$, the proof is completed.

(Step 3)

Let us now observe that

$$
\sum_{n=1}^{\infty} \alpha_{n}(\eta)^{2} n^{2 s-1} \geqq \text { const. }\langle\eta\rangle^{2 s}
$$

for $\eta$ contained in some conic neighborhood $F_{3}\left(\Subset F_{1}\right)$ of $\eta^{0}$ with $|\eta| \geqq 1$. Taking $\lambda(\eta) \in S_{1,0}^{0}\left(\boldsymbol{R}^{n_{2}}\right)$ with supp $\lambda \Subset F_{3}$ and $\lambda=1$ on $F_{4} \cap\{|\eta| \geqq 2\}$, we see that

$$
\lambda\left(D_{y}\right)(\beta v) \in H^{\infty},
$$

where $F_{4}$ is a small cone in $\boldsymbol{R}^{n_{2}}$ such that $\eta^{0} \in F_{4}$ and $F_{4} \Subset F_{3}$. Indeed, from (2.19) and Lemma 2.2 we have for any $s>0$ 


$$
\begin{aligned}
&\left.\iint i \lambda(\eta)|| \hat{\beta v}(\xi, \eta)\right|^{2}\langle\eta\rangle^{2 s} d \xi d \eta \\
& \leqq \text { const. } \sum_{n=1}^{\infty}\left\|\alpha_{n} \beta v\right\|^{2} n^{2 s-1}+\iint_{|\eta| \leqq 1}|\lambda(\eta) \| \hat{\beta v}(\xi, \eta)|^{2}\langle\eta\rangle^{2 s} d \xi d \eta \\
&<\infty .
\end{aligned}
$$

Noticing that $|\xi| \leqq c|\eta|$ in the support of $\gamma(\xi, \eta)$, we obtain (2.20).

Now take $\gamma_{1}(\xi, \eta) \in S_{1,0}^{0}\left(\boldsymbol{R}^{n}\right)$ with $\gamma_{1} \Subset \gamma$ and $\gamma_{1}=1$ on $\Gamma_{3} \cap\left\{|\xi|^{2}+|\eta|^{2} \geqq 2\right\}$. Here $\Gamma_{3}$ is a small cone in $\boldsymbol{R}^{n}$ such that $\left(0, \eta^{0}\right) \in \Gamma_{3}$ and $\Gamma_{3} \Subset \Gamma_{1}$. Then we have

$$
\lambda\left(D_{y}\right) \gamma_{1}\left(D_{x}, D_{y}\right)\left(\phi_{2} u\right)=\gamma_{1} \lambda\left(\phi_{2} \gamma u\right)+\lambda \gamma_{1} \phi_{2}(1-\gamma) u,
$$

where $\phi_{2}(x, y)=\chi(x) \beta(y)$. Since $\gamma_{1} \Subset \gamma$, it follows that $\gamma_{1} \phi_{2}(1-\gamma) u \in H^{\infty}$. So from (2.20) we have $\lambda \gamma_{1}\left(\phi_{2} u\right) \in H^{\infty}$. This implies $z \notin W F u$ and the proof of Proposition 1.1 is completed.

\section{$\S 3$. Proof of Theorem 1-(ii)}

Throughout this section, we assume that $l<m$. Now we prepare the following proposition.

Proposition 3.1. Let $\tilde{z}=(\tilde{x}, \tilde{y} ; \tilde{\xi}, \tilde{\eta})$ be an arbitrary point in $T^{*}\left(\boldsymbol{R}^{n}\right)$ with $|\tilde{\xi}||\tilde{\eta}| \neq 0$. If (A.1)-(A.4) are satisfied, then $L$ is microhypoelliptic at $\tilde{z}$.

The proof of Theorem 1-(ii) is completed by combining Propositions 1.1 and 3.1. So we give the proof of Proposition 3.1. The main tool is the following Hörmander's result, which we refer to Theorems 3.8 and 3.12 in Chapter 10 of Kumano-go [2].

Lemma 3.2. Let $P$ be a differential operator in $\boldsymbol{R}^{n}$ of order $k$ and $\Sigma$ be a conic neighborhood of $z_{0} \in T^{*}\left(\boldsymbol{R}^{n}\right)$. Let $p(x, \xi)$ be the symbol of $P$. Assume that $p(x, \xi)$ satisfies the following conditions.

(B.1) It holds that

$$
|p(x, \xi)| \geqq C_{0}|\xi|^{k^{\prime}} \quad \text { in } \Sigma \cap\{|\xi| \geqq M\}
$$

for some constants $M>0, C_{0}>0$ and $k^{\prime} \leqq k$.

(B.2) There exists a constant $\delta<1$ such that

$$
\left|p_{(\nu)}^{(\mu)}(x, \xi) / p(x, \xi)\right| \leqq C_{\mu, \nu}|\xi|^{\delta|\nu|-|\mu|} \quad \text { in } \Sigma \cap\{|\xi| \geqq M\}
$$

for some constant $C_{\mu, \nu}>0$, any indices $\mu$ and $\nu$. Here $p_{(\nu)}^{(\mu)}$ denotes $\partial_{\xi}^{\mu} D_{x}^{\nu} p$.

Then $P$ is microhypoelliptic at $z_{0}$. 
From now on, we show that (A.1)-(A.4) lead us to (B.1) and (B.2) if $p$ is the symbol of $L$ and $z_{0}=(\tilde{x}, \tilde{y} ; \tilde{\xi}, \tilde{\eta})$ with $|\tilde{\xi}||\tilde{\eta}| \neq 0$. For the sake of simplicity, we restrict our consideration to the case where $x, y \equiv \boldsymbol{R}\left(n_{1}=n_{2}=1\right)$ since the argument in general case is similar. According to the notations used in Lemma 3.2 , we write $x_{1}, x_{2}, \xi_{1}, \xi_{2}$ instead of $x, y, \xi, \eta$ below. So the symbol of $L$ is

Moreover we have

$$
p(x, \xi)=a\left(x, \xi_{1}\right)+g\left(x_{1}\right) b\left(x, \xi_{2}\right) .
$$

$$
p_{(\nu)}^{(\mu)}(x, \xi)=a_{(\nu)}^{(\mu)}\left(x, \xi_{1}\right)+\sum_{\beta \leqq \nu}\left(\begin{array}{l}
\nu \\
\beta
\end{array}\right) g_{(\beta)}\left(x_{1}\right) b_{(\nu)-\beta)}^{(\mu)}\left(x, \xi_{2}\right) .
$$

In view of (A.1)-(A.3), (B.1) is satisfied with $k^{\prime}=2 l$ if we choose the conic neighborhood $\Sigma$ of $z_{0}$ such that $\left|\xi_{1}\right| \sim\left|\xi_{2}\right|$ in $\Sigma$. So we check (B.2). Since $\left|\xi_{1}\right| \sim\left|\xi_{2}\right|$ in $\Sigma$, we get

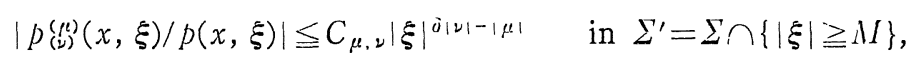

when $\nu=0$. Here $M$ is a large constant. Now, consider the case $\nu \neq 0$. In this case, we have

$$
\left|p_{(\nu)}^{(\mu)} / p\right| \leqq B|\xi|^{-|\mu|}\left(1+\sum_{\substack{\beta \leq \nu \\ \beta-0}} \min \left\{\left|g_{(\beta)}\left(x_{1}\right) g\left(x_{1}\right)^{-1}\right|,\left|g_{(\beta)}\left(x_{1}\right)\right| \xi_{1}^{2 J}\right\}\right)
$$

in $\Sigma^{\prime}$, where $j=m-l$ and $B$ is a large constant. From (A.4) we have

$$
\left|p_{(\nu)}^{(\mu)} / p\right| \leqq B|\xi|^{-\prime \prime}\left(1+C \sum_{\substack{\beta=\nu \\ 0}} \min \left\{g\left(x_{1}\right)^{-\tau \mid, S_{1}}, g\left(x_{1}\right)^{\left.1-\tau||^{\beta} \mid \xi_{1}^{2 \nu}\right\}}\right)\right.
$$

in $\Sigma^{\prime}$, when $|\nu| \leqq 2 j$. On the other hand, we see that

$$
\begin{aligned}
& g\left(x_{1}\right) \xi_{1}^{2 J} \leqq 1 \Longrightarrow g\left(x_{1}\right)^{1-\tau|\beta|} \xi_{1}^{2 J} \leqq\left|\xi_{1}\right|^{2 J \tau|\beta|}, \\
& g\left(x_{1}\right) \xi_{1}^{2 \jmath} \geqq 1 \Longrightarrow g\left(x_{1}\right)^{-\tau|\beta|} \leqq\left|\xi_{1}\right|^{2 J \tau|\beta|} .
\end{aligned}
$$

Thus it follows that

$$
\left|p_{(\nu)}^{(\mu)} / p\right| \leqq C_{\mu \nu}|\xi|^{\delta|\nu|-\mid \mu^{\prime \prime}} \quad \text { in } \nu^{\prime \prime}
$$

with $\delta=2 j \tau$, when $|\nu| \leqq 2 j$. When $|\nu|>2 j$, we have the above estimate with $\delta=2 j /(2 j+1)$. Since $\max (2 j \tau, 2 j /(2 j+1))<1$, we see that (B.2) holds.

Now in view of Lemma 3.2, the proof of Proposition 3.1 is completed.

\section{References}

L1] Fediř, V.S., On a criterion for hypoellipticity, Math. USSR Sb., 14 (1971), 15-45.

[2] Kumano-go, H., Pseudo-differential operators, M.I.T. Press, 1981.

[3] Morimoto, Y., On the hypoellipticity for infinitely degenerate semi-elliptic operators, J. Math. Soc. Japan, 30 (1978), 327-358. 
[4] Morimoto, Y. and Morioka, T., Some remarks on hypoelliptic operators which are not microhypoelliptic, in progress.

[5] Morioka, T., Hypoellipticity for semi-elliptic operators which degenerate on hypersurface, Osaka J. Math., 28 (1991), 563-578.

[6] Parenti, C. and Rodino, L., Examples of hypoelliptic operators which are not microhypoelliptic, Boll. Un. Mat., 17-B (1980), 390-409. 